\title{
Study of the Position of Sound Sources in a Turbulent Jet Using Nozzles of Different Configurations
}

\author{
Victor Ershov* and Igor K hramtsov \\ Perm N ational Research Polytechnic University, 614013 Perm, Russian Federation
}

\begin{abstract}
The aim of this work is to study the position of dominant sound sources in a small-scale turbulent jet using the beamforming method. Two nozzles of equivalent diameter and different geometric configurations (conical and chevron) were used to create different initial conditions for the outflow. Based on the analysis of the results obtained, it can be concluded that they are in good agreement with the well-known concepts of the physics of noise generation processes by turbulent jets: higher-frequency sources are generated by smaller-scale turbulent structures located closer to the nozzle edge, which is confirmed by the localization of high-frequency noise sources also closer to the nozzle edge. The chevron nozzles loosen the initial section of the jet, making it less short and thereby facilitating a faster displacement of noise sources to the nozzle edge, as seen when comparing localization maps for conical and chevron nozzles at the same frequencies. The results of localization were compared with the data obtained by other researchers. The results obtained were found to provide confidence in the use of acoustic beamforming to measure the location of the jet noise source with accuracy similar to other methods that have been used in the past.
\end{abstract}

\section{Introduction}

The formulation and methods of solving modern problems to reduce the negative impact of air transport on the environment and humanity are initiated by the International Civil Aviation Organization (ICAO). To date, research in the field of aircraft noise abatement has achieved significant results. However, the main source in the general aircraft noise is still the engine [1]. In the noise of the aircraft engine itself, one of the main sources is a turbulent jet, which is due to the following reasons. First, advances in research on noise reduction of bladed machines have led to the fact that even in engines with a high bypass ratio, the jet has a sufficiently high level of noise emission. Secondly, in practice, even for modern passenger aircraft, engines with a low bypass ratio are often used, in which the noise of the jet stream makes the main contribution to the overall noise of the power plant.

As is known, there are active and passive methods of influencing turbulence, and, consequently, the noise generated by a turbulent jet. The methods of active control include the impact of acoustic or vibration disturbances on the jet nozzle $[2,3]$, as well as the impact

* Corresponding author: er.vittore@ gmail.com 
of plasma actuators [3-6] and injection of microjets into the main stream [7-12]. In such cases, by choosing the excitation frequency, it is possible to enhance or weaken the merging of vortex structures, and, therefore, to influence the jet noise. Passive control is carried out by changing the flow conditions or changing the geometry of the jet-forming device. In this case, mixing is mainly intensified, which changes the properties of large-scale vortex structures and reduces the length of the initial section of the jet. These methods include slit (rectangular), chevron, beveled and corrugated nozzles [2, 13-18]. These methods are among the most effective (noise reduction up to $5 \mathrm{~dB}$ ). In addition, they are simple - usually no additional hardware is required, and their use does not increase the mass of the aircraft engine.

Chevron nozzles are most often used to reduce the jet noise of real aircraft engines. This form is the most common, since it combines ease of manufacture and a fairly high efficiency (up to $3-5 \mathrm{~dB}$ ). The use of such nozzles makes it possible to reduce the jet noise in the region of the maximum in the noise spectrum, but at the same time, there is an increase in noise in the high-frequency region of the spectrum. However, from the point of view of assessing and predicting aircraft noise on the ground, it is more important to reduce the noise precisely in the area of maximum spectrum. Greater efficiency of chevron nozzles is achieved by increasing the size of the petals and a larger deflection angle of the chevrons into the jet stream. However, striving for greater efficiency in noise reduction leads to a loss of engine thrust up to several percent [18].

The location of noise sources is critical information. This is especially true for the noise of a turbulent jet, in which the noise is generated by many vortices of various scales interacting with each other. This leads to the fact that noise sources at different frequencies are distributed within the jet. Since chevrons lead to a change in large-scale eddies, accordingly, this should lead to a change in the position of noise sources from a given object relative to the jet flowing from a standard nozzle.

In this work, a planar microphone array was used to obtain information on the sources of noise in the jet, the measurement signals from which were processed by the planar beamforming method. Using this method, it is possible to obtain information on the distribution of acoustic sources in space, to determine the sound pressure level of these sources at frequencies of interest. Beamforming methods are widely used in aeroacoustic research [19-25].

The main idea of the method is to coherently sum the measurement results of different microphones to improve the signal emitted from the focal point and minimize the contribution of signals from all other points. Thus, the beamforming method is a method of adding data measured by microphones in order to determine the spatial distribution of noise sources. In the simplest case of the method, the results of microphones measurements are added with a time delay, where the amount of delay depends on the focus position and the position of the microphone. When the sound source is at the focal point, the microphone measurements are added coherently, resulting in amplification of the signal, and incoherently when the source is out of focus. The method of beamforming has proven itself well for localizing isolated point sound sources [24].

\section{Experimental study of jet noise with standard and chevron nozzles}

The work considered standard conical and chevron nozzles. The conical nozzle is a 12-degree taper and has a sharp cut edge. A detailed study of the noise of a jet outflowing from the used conical nozzles is considered in [26, 27]. The chevron nozzle used in this work had 12 chevrons located along the nozzle output contour [28]. In this case, the angle of deflection of the chevrons into the jet remains the same as that of the conical nozzle. The equivalent area along the chevrons midline corresponds to the conical nozzle output area. A distinctive 
feature of these chevrons is the lateral triangular shape of the chevron and the sharp edges of the chevrons (Fig. 1).
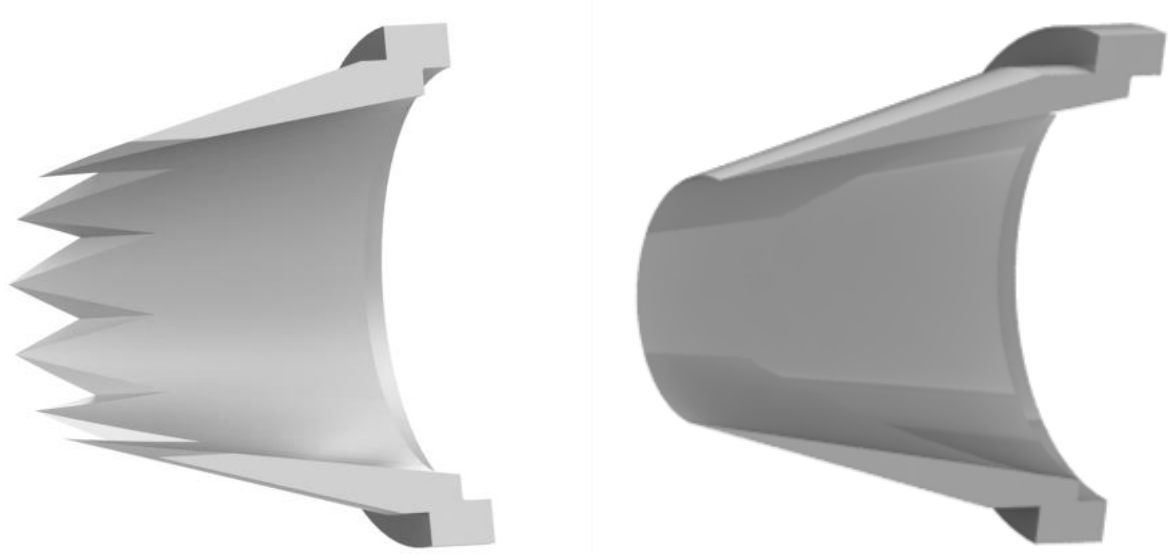

Fig. 1. 3D models of chevron (left) and conical (right) nozzles.

The chevron nozzles were manufactured in two stages. At the first stage, a conical nozzle was turned using a CNC machine, which was further subjected to additional processing. There is a sharp edge in the nozzle output area, and the use of traditional machining methods based on mechanical influences leads to its deformation. In this case, laser cutting of metal was used to cut the chevrons. As a result, sharp edged chevrons were obtained.

Studies of a single-stream jet were carried out in an anechoic chamber in PNRPU [2931]. The authors used a single-stream jet without cocurrent flow at a speed of $0.5 \mathrm{M}$, created using two series-connected fans, each with a power of $45 \mathrm{~kW}$. The speed was controlled before the experiment using a Pitot-Prandtl tube. The nozzle size was $50 \mathrm{~mm}$. When measuring the noise of the jet, the microphones were installed in the plane of the jet axis parallel to the floor on free-standing racks. To reduce reflections from the racks, the microphones were attached through special holders $0.4 \mathrm{~m}$ long. The racks were positioned so that the receiving microphone heads were on an arc with a radius of $2 \mathrm{~m}$ from the center of the nozzle exit at an angle of 30 to 105 degrees relative to the nozzle axis directed towards the jet outflow. The racks were located in 15-degree increments. Signal recording in each measurement was performed using 1/4" Bruel \& Kjaer microphones, type 4958 for $10 \mathrm{~s}$. Bruel \& Kjaer analyzers, type 3055-B-120, and specialized Bruel \& Kjaer PULSE LabShop software were used for recording. A photograph of the experiment in the PNRPU anechoic chamber is shown in Figure 2. The obtained noise spectra of the jet generated by the conical and chevron nozzles for observation angles of 90 and 30 degrees are shown in Figure 3. 

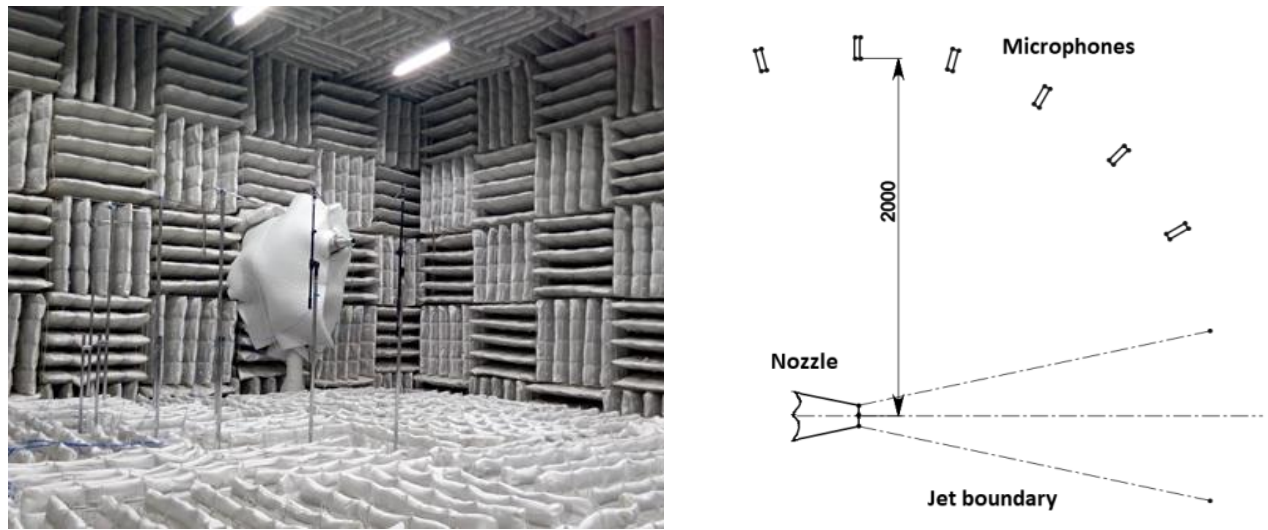

Fig. 2. Photo and scheme of the experiment for measuring the noise of a jet with different nozzles.
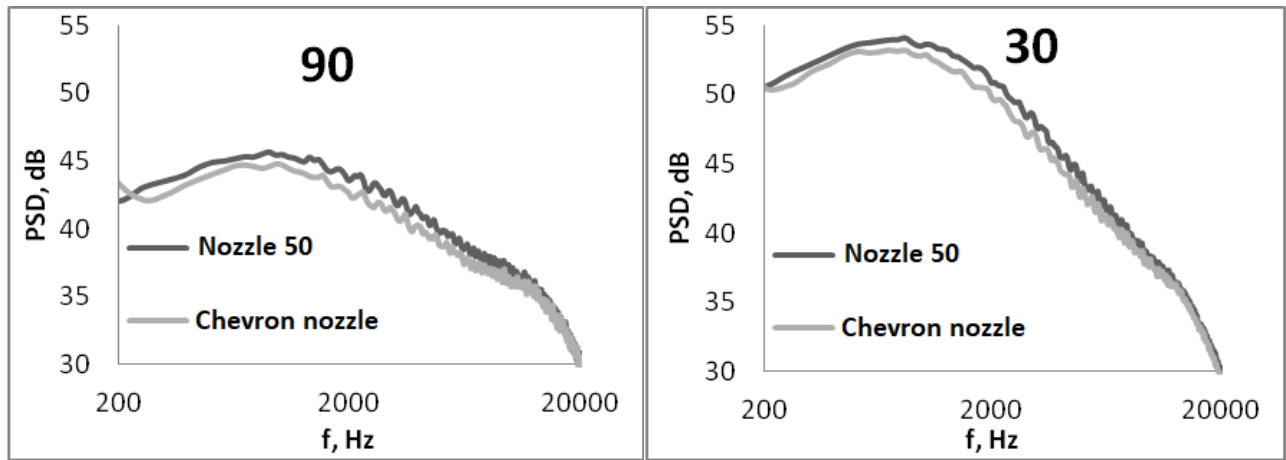

Fig. 3. Noise spectra of chevron nozzle in far field.

As can be seen, the chevron nozzles provide a noise reduction efficiency of up to $1 \mathrm{~dB}$ at the peak of the jet noise, with a slight increase in the high frequency noise component.

\section{Experimental study of the position of noise sources of a turbulent jet}

To localize noise sources in turbulent jets, a Bruel \& Kjaer WA-1676-W-003 9-beam 54microphone array was used, in which Bruel \& Kjaer 4944 microphones were installed. The jet noise was recorded and excitation was generated using the Pulse LabShop NSI Array Fixed Meas application. The noise was recorded for $10 \mathrm{~s}$. Investigations of the position of noise sources by the beamforming method were carried out under similar conditions of jet outflow. The jet velocity was $0.5 \mathrm{M}$. A conical and chevron nozzle was used. The microphone array was installed so that the center of the array was opposite the nozzle output, and the jet axis crossed the center of the localization map. The array was removed from the plane of the jet at a distance equivalent to 1 diameter of its imaginary circumscribed circle. Figure 4 shows the layout of the microphone array and a photograph of the experiment. 


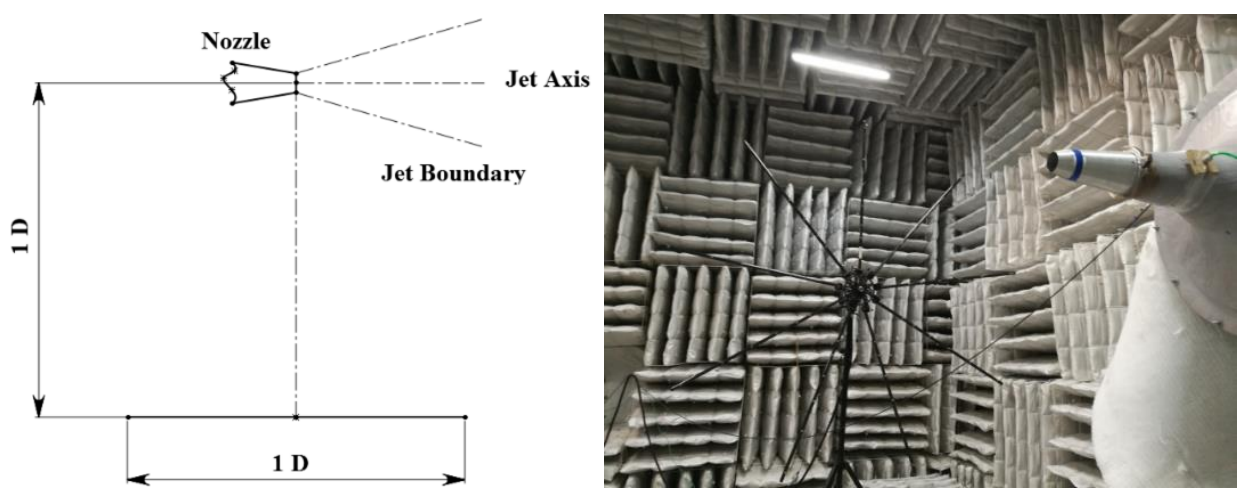

Fig. 4. The layout of the microphone array and a photograph of the experiment.

The results were processed using the NSI Array Acoustic Post-Processing program using the Beamforming Delay-and-Sum method. The results were analyzed in one-third octave frequency bands from 315 to $20,000 \mathrm{~Hz}$. When constructing localization maps, a dynamic range of $3 \mathrm{~dB}$ was used. The sources were localized on a sufficiently detailed grid with a linear element size of $1 \mathrm{~cm}$. At the first stage, the source position patterns were compared at different frequencies for jets obtained using conical and chevron nozzles.

For each frequency band, an image with a source localization map was saved. A 50\% overlap was applied to the sequence procedure for selecting data blocks for processing. Each block was multiplied by a Hann window function, then a Fast Fourier Transform was performed. The number of FFT spectral lines was 400, which gives the width of each spectral line with a frequency of $64 \mathrm{~Hz}$. From each spectral sample, applied to all microphones in the array, cross-spectral matrices were generated, corresponding to each specific frequency. After digital processing of all data blocks in the original record, a linear averaging of the entire set of cross-spectral matrices related to the same spectral samples was carried out.

After performing the post-processing procedure, the location of the acoustic sources with the maximum radiation intensity was determined for each one-third octave frequency band. Based on the information about the coordinates of the nozzle output obtained using the visualization map, the distance from the exit to each of such sources was calculated. At the first stage, a comparison was made of the source position patterns at different frequencies for jets obtained using nozzles of different diameters.

As can be seen in Fig. 5, for a jet formed with a conical nozzle, characteristic manifestations of this type of flow are observed, in particular, with increasing frequency, the source decreases and shifts closer to the nozzle output. For a jet formed with a chevron nozzle, the position of the sources is close to the position in a clean jet, however, with increasing frequency, the source moves faster closer to the nozzle output. As can be seen in the figure, the center of the source in the area of the maximum jet noise for a given speed $(500-2000 \mathrm{~Hz})$ is located at a distance of 4-9 calibers from the nozzle output, which corresponds to the end of the initial section and the beginning of the main section in the buoyant jet [32]. 


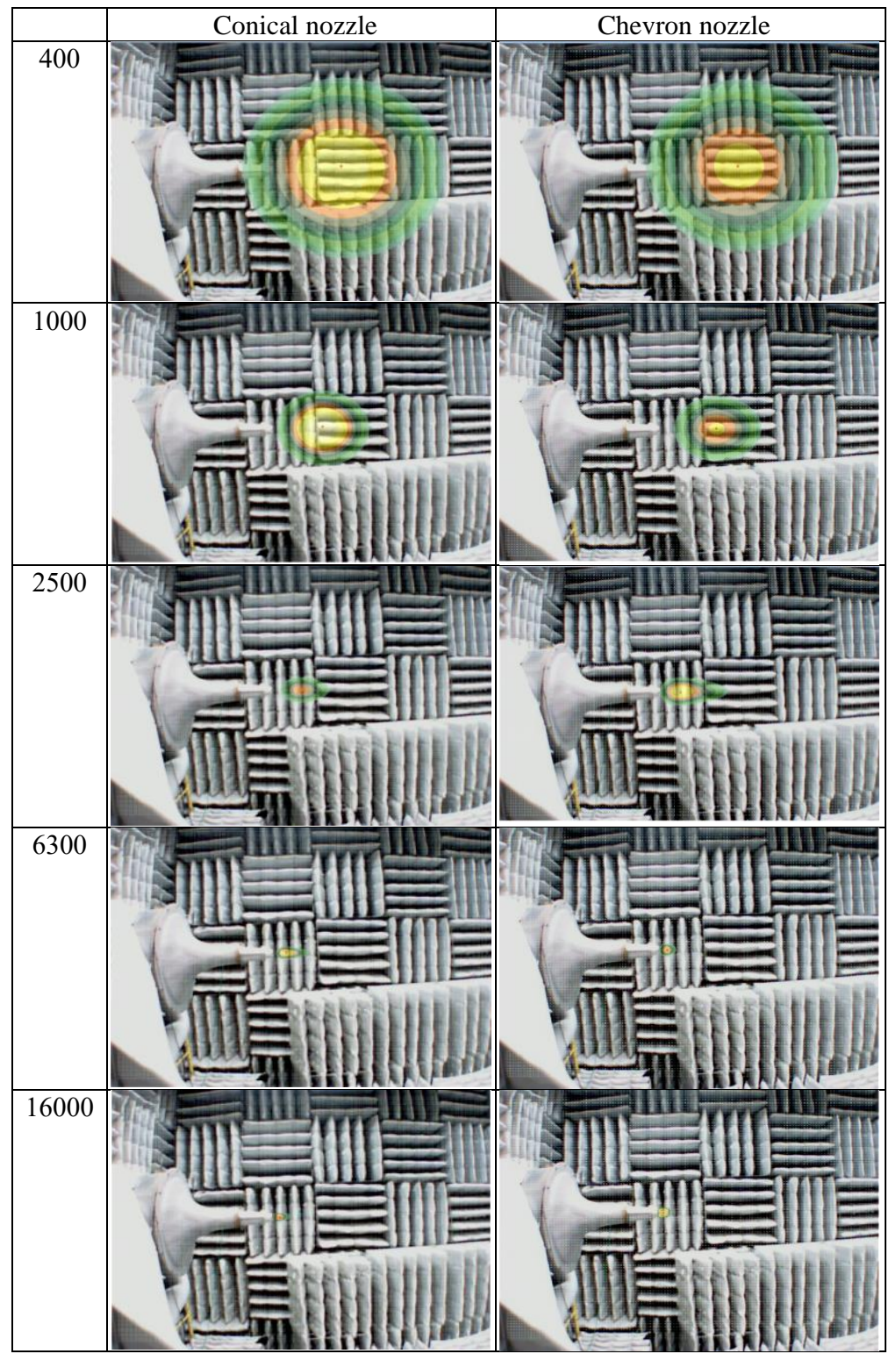

Fig. 5. Localization map of noise sources in a clean jet.

Subsequently, a more detailed analysis of the position of the source of a clean jet was carried out in accordance with [22]. In this work, the position of the center of the noise source on the localization map for turbulent jets was considered. In this case, the position of noise sources at different frequencies is concentrated near:

$$
S t=50(x / d \mathrm{c})-2.5
$$

where $S t$ is the Strouhal number; $x$ - the position of the center of the noise source on the localization map; $d_{\mathrm{c}}$ - nozzle diameter.

It is also shown in this work that the position of the sources depends on the Mach number, Reynolds number, turbulence in the initial section, etc. The results obtained in this study were 
compared with expression (1) and the results of other authors, in whose works the following methods were used: acoustic mirror from Chu et al. [33], polar correlation technique from Fisher et al. [34] and the minimum phase from Ahuja et al. [35].

Figure 7 shows a typical comparison of the results obtained in this work with the results of various author for a conical nozzle with a diameter of $50 \mathrm{~mm}$. It can be seen that the results obtained using the Bruel \& Kjaer array are quite close to other data and methods, however, in the presented works, there is a significant scatter of the initial outflow conditions (nozzle size, jet outflow velocity, etc.).

As can be seen in Fig. 6, the results obtained by the beamforming method are in good agreement with the results of [22], in which a similar method was used. The positions of the noise sources under similar outflow conditions practically coincide with each other for different jet installations. The differences in the position of the source at close frequencies are no more than 1 caliber.

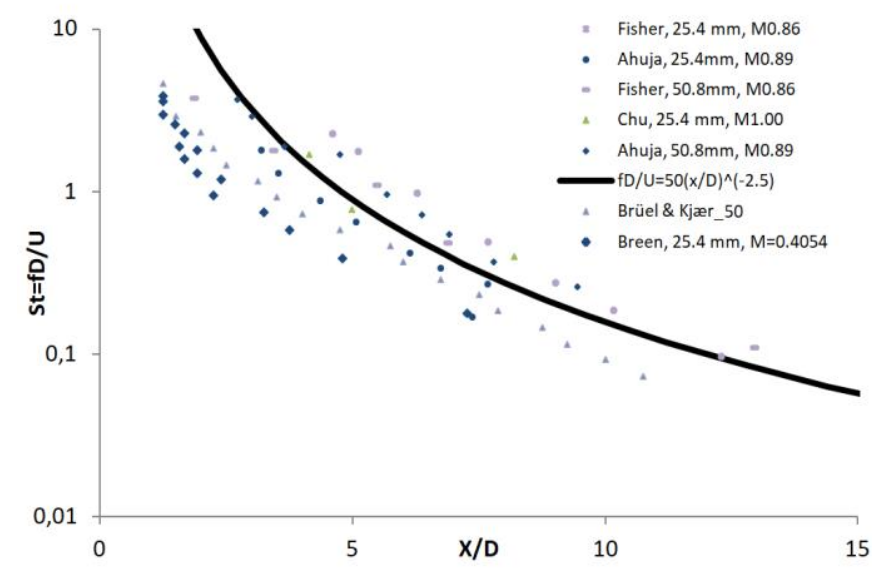

Fig. 6. Comparison of the results by the position of the noise source with other authors.

It should be noted that the most reliable is the comparison of the results of determining the location of the source obtained using different methods on the same setup under the same outflow conditions [22].

Fig. 7 shows a comparison of noise sources position in the jet flowing from the conical and chevron nozzles. As can be seen, the noise sources positions in the chevron jet at high frequencies are shifted closer to the nozzle output, which is associated with the refinement of the size of the vortices that are formed on the chevron lobes due to their azimuthal inhomogeneity.

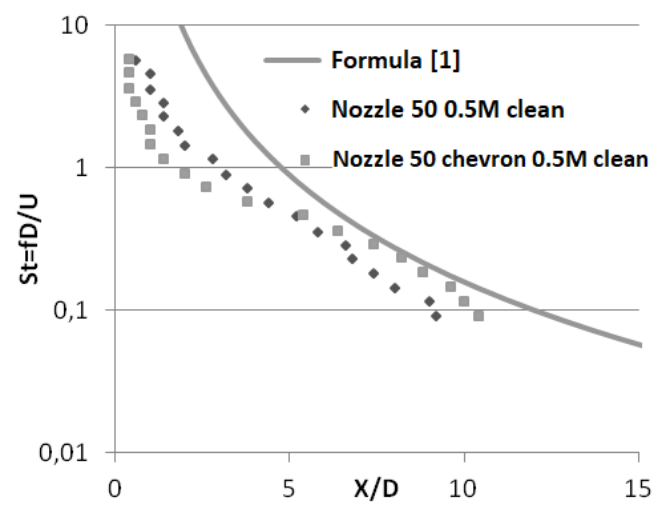

Fig. 7. Dependence of source position on localization maps for conical and chevron nozzles. 


\section{Conclusion}

This paper considers the acoustic characteristics of jets emanating from a conical and chevron nozzle. Metal chevron nozzles are used with equivalent dimensions corresponding to a conical nozzle with an outlet diameter of $50 \mathrm{~mm}$. These chevron nozzles have 12 triangular chevrons located along the contour of the nozzle output. The experiments carried out to measure the noise of a turbulent jet showed that the efficiency of the created chevron nozzles in the region of the radiation maximum is about $1 \mathrm{~dB}$. In this case, the high-frequency part of the spectrum increases slightly. For assessing and predicting aircraft noise on the ground, it is more important to reduce noise precisely in the area of maximum radiation.

Localization is carried out of noise sources in a turbulent jet formed with conical and chevron nozzles. A Bruel \& Kjaer 9-beam 54-channel planar microphone array and Beamforming Delay-and-Sum method were used. As a result, the position of noise sources in a turbulent jet in one-third octave frequency bands was obtained.

The results obtained for the jet outflowing from the conical nozzle were compared with the results of other authors and the expression for the Strouhal number from [22]. It was found that the position of the noise sources in the jet is close enough to the calculated one. The differences can be explained by the difference in the initial conditions of the jet outflow. Comparisons of the positions of the noise sources in the jet flowing from the conical and chevron nozzles showed that at high frequencies the source is located closer to the nozzle exit.

This work was supported by JSC «United Engine Corporation».

\section{References}

1. L. Leylekian, M. Lebrun, P. Lempereur, J. A erospace Lab, 7, 1-15 (2014).

2. V.M. K uznetsov. Fundamentals of the theory of turbulent jet noise, 240 (2008) [In Russian]

3. V.F. Kopiev, I.V. B elyaev, G.A. Faranosov, V.A. Kopiev, N.N. Ostrikov, M.Y u. Zaytsev, Y u.S. A kishev, M .E. Grushin, N.I. Trushkin, V .A. Bityurin, A.I. Klimov, I.A. M oralev, I.A. K ossyi, N .K. B erezhetskaya, M .I. Taktakishvili, Progress in Flight Physics, 7, 211-228 (2015)

4. J.H. K im, M. Kearney-Fischer, M. Samimy, S. Gogineni, A IA A Paper, 3187, 2009.

5. V.F. Kopiev, V.A. Bityurin, I. V. B elyaev et al. A coustical Physics 58, 473-481. (2012) [In Russian]

6. V.F. Kopiev, Y u.S. A kishev, I.V. B elyaev, N.K. B erezhetskaya, V .A. Bityurin, G.A. Faranosov, M .E. Grushin, A.I. Klimov, V .A. Kopiev, I.A. Kossyi, I.A. M oralev, N.N. Ostrikov, M .I. Taktakishvili., N .I. Trushkin, M .Y u. Zaytsev, J . of Physics D: A pplied Physics 47, 1-18 (2014)

7. E. Zoppellari, D. J uve, A IA A Paper 2204 (1998)

8. A. K rothapalli, B. Greska, V. A rakeri, A IA A Paper 2450 (2002)

9. B. Greska, A. K rothapalli, A IA A Paper 3046 (2005)

10. T. Castelain, M. Sunyach, D. Juve, J.C. Bera, A IA A Paper 3419 (2007)

11. K.B.M.Q. Zaman, G.G. Podboy, A IA A Paper 4022 (2010)

12. D.A. Gubanov, Influence of microjets on the structure and acoustic radiation of a supersonic underexpanded jet. Thesis for the Ph.D. degree (Phys. and M ath.), 140 (2014) [In Russian] 
13. F. Geren, A trailing edge for an aircraft engine provided with moving chevron elements and an aircraft nacelle provided with such a trailing edge. Patent 2492337 (2013) [In Russian]

14. A.A. Silla, J. Hubert, O. Pelagatti, D. Pra, and K. Debatin, A nti-noise chevron for a nozzle, a nozzle and a turbojet equipped with such a chevron. Patent 2466290 (2012) [In Russian]

15. A.A. A leksentsev, D.B. B ekurin, E.V. V lasov, et. all, . TsA GI Scientific Records XL, 14-21 (2009) [In Russian]

16. D.A. L yubimov, A nalysis of turbulent jet and separated flows in turbojet engine elements by combined RANS / LES methods of high resolution. Thesis for the Ph.D. degree (Phys. and M ath.), 40 (2014) [In Russian]

17. V.F. Kopiev, M.Y u. Zaitsev, N.N. Ostrikov, A coustical Physics 59, 232-234 (2013) [In Russian]

18. A.A . A leksentsev, D.B. B ekurin, E.V. V lasov, et. all, . TsA GI Scientific Records XL, 14-21 (2009) [In Russian]

19. S. Gade, J. Hald, J. Gomes, G.Dirks, B. Ginn, Sound \& V ibration 49, 8-14 (2015)

20. J.J.Christensen, J. Hald B eamforming. Technical review. Brüel \& Kjær Sound \& Vibration M easurements A/S (2004)

21. R.P. Dougherty, A eroacoustic M easurements, 62-97 (2002)

22. N. P. B reen and K. A huja, A IA A Paper 2015-0735 (2015).

23. V.F. Kopiev, I.V. K hramtsov, V.V. Ershov, V.V. Palchikovskiy, A coustical Physics 65, 67-75 (2019)

24. Y u.V. Bersenev, T .A . V iskova, I.V. B elyaev, V.V. Palchikovskiy, O.Y u. K ustov, V.V. Ershov, R.V. Burdakov, PNRPU M echanics Bulletin 1, 26-38 (2016).

25. O.P. Bychkov, M.A. Demyanov and G.A. Faranosov, A coustical Physics 65, 567-577 (2019).

26. I.V. K hramtsov, E.S. Cherenkova, V.V. Palchikovskiy, O.Y u. K ustov, A IP Conference Proceedings 1770, (2016).

27. I.V. K hramtsov, E.S. Cherenkova, V.V. Palchikovskiy, O.Y u. K ustov, AKUSTIKA 34, 180-184 (2019).

28. I.V. K hramtsov, E.S. Cherenkova, V.V. Palchikovskiy, O.Y u. K ustov, A erospace Engineering, High Technologies and Innovation 1, 285-288 (2017).

29. V.F. Kopiev, V.V. Palchikovskiy, I.V. B elyaev, Y u.V. B ersenev, S.Y u. M akashov, I.V. K hramtsov, I.A. Korin, E.V. Sorokin, O.Y u. K ustov, A coustical Physics 63, 113124 (2017).

30. V.V. Palchikovskiy, Y u.V. B ersenev, S.Y u. M akashov, I.V. B elyaev, I.A. K orin, E.V. Sorokin, I.V. K hramtsov, O.Y u. K ustov, A IP Conference Proceedings 1770 (2016).

31. V. F. Kopiev, S. Y u. M akashov, I. V . Belyaev, V. V. Palchikovskiy, Y u. V. B ersenev, I. A. K orin, E.V. Sorokin, I.V. K hramtsov and O.Y u. K ustov, Procedia Engineering 176 (2017).

32. G.N. A bramovich, The theory of turbulent jets. 720 (2011) [In Russian]

33. W. T. Chu, J. Laufer, K. K ao, Noise Source Distribution in Subsonic Jets. International Conference on N oise Control, W ashington D.C. (1972).

34. M.J . Fisher, M. H arper-B ourne, S.A.L. Glegg, J. Sound and V ibration 51, 23-54 (1977)

35. K.K. Ahuja, K.C. Massey, M.S. D’Agostino, AIAA Paper 98-2359 (1998) 